\title{
Identificación de factores predictores de técnica epidural dificultosa en la paciente obstétrica
}

\author{
L. M. Charco Roca 1 , V. E. Ortiz Sánchez ${ }^{1}$, P. Cuesta Montero ${ }^{2}$, A. Soria Quiles ${ }^{1}$ y L. Bonmati García ${ }^{1}$ \\ ${ }^{I}$ Servicio de Anestesiología y Reanimación. Hospital Virgen del Castillo. Yecla \\ ${ }^{2}$ Servicio de Anestesiología y Reanimación. Complejo Hospitalario Universitario de Albacete
}

L. M. Charco Roca, V. E. Ortiz Sánchez, P. Cuesta Montero, A. Soria Quiles y L. Bonmati García. Identificación de factores predictores de técnica epidural dificultosa en la paciente obstétrica. Rev Soc Esp Dolor 2013; 20(5): 216-220.

\begin{abstract}
Introduction: Repeated attempts punctureare risk factors for the appearance of neurologic complications caused by the practice of spinal analgesia and increase patient anxiety. The purpose of this study is to determine which factor sare better predictors of difficult epidural catheter placement in the obstetric patient.

Material and methods: Prospective observational study in 120 pregnant women at term who request edepidural analgesia during labor. Demographic variables and history of difficult or unsuccessful spinal anesthesia were collected. Anatomical variables were identified and the quality of anatomical landmarks was classified according to criteria published by Chien in four grades. The conditions of the technique and the experience of anesthesiologists were similar in all cases. It was considered as difficult puncture that in which was made more than one attempt on skin or more than one change of direction with the epidural needle in interspinous space.We collected the successand complications of the technique.

Results: $36.67 \%$ of cases were classified as difficult punctures according to the criteria of the study. In most cases the technique was effective obtaining asuccessful analgesia. The incidence of repeat epidural puncture was $5 \%$. In patients classified in grade 4 , the percentage of difficult epidural puncture was $90 \%$, requiring in $80 \%$ of the cases 3 or more attempts. In patients with a history of difficult neuraxial technique in $28.57 \%$ of the cases was found difficulty in epidural puncture. Body habitus had no significant effect on the rate success in first attempt.
\end{abstract}

Recibido: 06-04-12.

Aceptado: 15-07-12
Discussion: Our study represents a good estimationof a difficult neuraxial blockade. It would bedifficult to know if there flected datawould be applicable to the lateral decubitus technique where anatomical landmarksare more difficult to identify.

Conclusions: Our study concludes thatt he most reliable method to predict the possibility of a technical difficulty in the placement of lumbar epidural catheter, is the examination of the back of the patient to identify the quality of anatomical landmarks and identify obvious deformities of the spine.

Key words: Obstetric analgesia. Epidural analgesia. Neuroaxial block.

\section{RESUMEN}

Introducción: Los intentos repetidos de punción son factores de riesgo para la aparición de complicaciones neurológicas secundarias a la práctica de la analgesia espinal y aumentan la ansiedad del paciente. El objetivo de este estudio es determinar qué factores son mejores predictores de la colocación dificultosa del catéter epidural en la paciente obstétrica.

Material y método: Estudio observacional y prospectivo en 120 gestantes a término que solicitan la analgesia epidural para el trabajo de parto. Se recogen variables demográficas y antecedentes de anestesia espinal previa no exitosa o considerada dificultosa por la paciente. Se identifican variables anatómicas y se clasifica la calidad de los puntos de referencia anatómicos según los criterios publicados por Chien en cuatro grados. Las condiciones de la técnica y la experiencia del anestesiólogo son similares en todos casos. Consideramos punción dificultosa si se necesita más de una punción en la piel o una punción pero más de un cambio de dirección de aguja en el espacio interespinoso. Se recoge el éxito obtenido y las complicaciones de la técnica.

Resultados: Fueron calificadas de punción difícil según los criterios del estudio en el 36,67 \% de los casos. En la mayoría de los casos la técnica fue efectiva obteniendo una adecuada analgesia. La incidencia de repunción epidural fue de $5 \%$. En las pacientes clasificadas en el grado 4 , la tasa de punción epi- 
dural considerada dificultosa supuso un $90 \%$, necesitando en el $80 \%$ de esos casos 3 o más intentos. En las pacientes con antecedentes personales de técnica neuroaxial dificultosa se encontró dificultad de punción epidural en un 28,57 \% de los casos. El hábito corporal no tuvo efecto significativo sobre la tasa de éxito con el primer intento.

Discusión: Nuestro estudio representa una buena estimación de la dificultad técnica de un bloqueo neuroaxial. Seria difícil saber si los datos reflejados serían extrapolables a la realización de la técnica en decúbito lateral, donde los puntos de referencia anatómicos son más difíciles de identificar.

Conclusiones: Nuestra recogida concluye que el método más fiable para determinar de antemano la posibilidad de una dificultad técnica de colocación del catéter epidural lumbar es un examen de la espalda de la paciente para identificar la calidad de los puntos de referencia anatómicos e identificar la deformidad obvia de la columna vertebral.

Palabras clave: Analgesia obstétrica. Analgesia epidural. Bloqueo neuroaxial.

\section{INTRODUCCIÓN}

La analgesia epidural se utiliza ampliamente en nuestro país para el alivio del dolor del parto. Sabemos que el dolor tiene como objetivo avisar a la gestante de que el parto se ha iniciado pero, una vez cumplida esa misión, el dolor no tiene ninguna utilidad. No hay evidencia de que el dolor del parto sea beneficioso para la madre o el feto, sino que puede tener repercusiones negativas sobre el proceso; es un poderoso estímulo ventilatorio que provoca hiperventilación materna y conduce a alcalosis respiratoria. A su vez, el dolor, unido a la ansiedad y al aumento de la actividad física materna, condicionan un aumento del gasto cardiaco por estímulo simpático, un aumento de la tensión arterial, un incremento del metabolismo aeróbico y anaeróbico de los hidratos de carbono con aumento del ácido láctico materno y un aumento del consumo de oxígeno. La hiperactividad adrenérgica ocasiona una disminución del flujo sanguíneo uterino por vasoconstricción uteroplacentaria e incoordinación de la actividad uterina. Finalmente, el dolor y la ansiedad también provocan una disminución de la motilidad gastrointestinal con retardo del vaciado gástrico y con el consiguiente aumento del riesgo de aspiración pulmonar si es precisa la realización de una anestesia general.

Ha habido un interés creciente de nuevas generaciones de anestesiólogos por las técnicas regionales, que, unido al cambio de mentalidad en la mujer actual, que exige no solo que la asistencia sea correcta sino poder tener una mayor participación en el momento del parto, y que quiere estar consciente y compartir con su pareja este momento y comprobar personalmente lo que ocurre con su hijo, han hecho de la analgesia epidural la técnica más extendida para el alivio del dolor del parto y actualmente constituye una de las prácticas mas solicitadas al anestesiólogo. La paciente obstétrica con trabajo de parto se diferencia de otros pacientes que podamos encontrar en la práctica clínica habitual por su elevado grado de ansiedad que acompaña a las contracciones uterinas dolorosas, lo que dificulta o imposibilita la colocación de un catéter en espacio epidural por los continuos movimientos de la paciente. Es deseable en todos los casos en que practicamos anestesia regional, y aun más en la paciente obstétrica, evitar realizar técnicas prolongadas y dolorosas que aumenten la situación de ansiedad, y sería en sí misma una razón para tratar de prever una dificultad en la punción epidural antes de comenzar. Por otra parte, los intentos repetidos de punción o la presentación de pacientes con deformidades espinales evidentes a la inspección son considerados factores de riesgo para la aparición de complicaciones neurológicas secundarias a la práctica de la analgesia espinal, especialmente en lo referente a lesiones traumáticas de la médula o de la cola de caballo, como consecuencia de punciones en niveles inadecuados, ya sea por inexperiencia, equivocación o dificultad para la punción en otros espacios $(1,2)$. Algunos autores han sugerido que, ante una espalda en la que se identifiquen factores predictores de dificultad de punción, se busque al anestesiólogo más experto para evitar, en lo posible, complicaciones (3).

En los hospitales universitarios se forman residentes de anestesiología para la práctica segura de procedimientos de bloqueo regional anestésico. Las curvas de aprendizaje publicadas hasta ahora muestran una tasa aceptable de fallos y complicaciones en las técnicas epidurales, y el número de procedimientos necesarios para considerar a un anestesiólogo adecuadamente preparado ha sido objeto de numerosos estudios $(4,5)$.

Realizar múltiples intentos de punción para la colocación del catéter y de identificación del espacio epidural pueden provocar molestias para la paciente, mayor incidencia de hematoma epidural, cefalea pospunción dural y el riesgo de trauma de las estructuras neuroaxiales. Hay factores que han sido identificados como predictores de un bloqueo neuroaxial técnicamente difícil (6-8).

El objetivo de este estudio es determinar qué factores de la paciente son mejores predictores de la colocación dificultosa del catéter epidural en la paciente obstétrica.

\section{MATERIAL Y MÉTODO}

Presentamos un estudio observacional y prospectivo que incluye 120 gestantes a término, clasificadas ASA I y II, que solicitan la analgesia epidural voluntariamente para el trabajo de parto en un hospital universitario durante un periodo de 13 meses; entre octubre de 2010 y noviembre de 2011. La recogida de los casos ha sido de forma no consecutiva y aleatoria. Durante este periodo de tiempo fueron realizadas en este centro 1.866 epidurales obstétricas. 
Previo a la realización de la técnica se informó a las pacientes acerca de la técnica que se iba a realizar (en todos los casos, epidural con fines analgésicos para el dolor de parto) y se solicitó la firma de un consentimiento escrito según el protocolo del centro hospitalario. Al tratarse de un hospital con formación de residentes, las pacientes aceptan con este escrito que sus datos sean utilizados y analizados con fines científicos siempre que esta premisa no suponga cambios en la actitud terapéutica.

Previo a la realización de la técnica fueron recogidas unas variables demográficas de la paciente: edad, peso, talla, IMC y hábito corporal, clasificadas como normales u obesas si el IMC es mayor de 30. Fueron recogidos asimismo mediante la entrevista clínica antecedentes de punción epidural o intradural previa no exitosa o considerada dificultosa por la paciente, según su experiencia subjetiva del proceso anterior; resultando positivo este punto, referían demasiado tiempo empleado en la técnica, recuerdos de considerarla dolorosa o demasiado incómoda, o insatisfacción con la calidad analgésica obtenida. Mediante inspección visual y palpación de la columna de la paciente, se identifican variables anatómicas sobre presencia o ausencia de signos de escoliosis, palpación de crestas iliacas o no, y se clasifica la calidad de los puntos de referencia anatómicos según los criterios publicados por Chien I. (8) en cuatro grados. Grado 1: cuando las apófisis espinosas son visibles; grado 2: si las apófisis espinosas no se ven, pero se palpan fácilmente; grado 3: cuando las apófisis espinosas no se ven ni se palpan, pero el intervalo entre ellas es palpable (posibilidad de hundir el pulgar en los espacios interespinosos lumbares); y grado 4: si no hay presentes ninguna de las referencias anteriores. En los casos de clasificadas en el grupo 4, la anatomía espinal es intuida y el punto de punción es seleccionado por el anestesista según inspección anatómica.

En cuanto a la técnica de punción, todas fueron realizadas con la gestante en posición sentada. La técnica fué realizada por residentes de anestesiología de $3-4^{\circ}$ año con experiencia previa de haber realizado al menos 120 epidurales (lumbares o torácicas). Se utiliza aguja Tuoy 18G y la punción se realiza a nivel L2, L3 o L4 (el mejor espacio identificado por el anestesiólogo). Consideramos una punción difícil si la identificación del espacio epidural y la colocación del catéter necesita más de una punción en la piel o una punción de la piel, pero más de un cambio de dirección de aguja en el espacio interespinoso. Tras la punción epidural fueron recogidos el número de intentos realizados, el anestésico utilizado y el resultado analgésico obtenido, considerándola exitosa si tras 20 minutos de la dosis inicial el dolor referido por la paciente era igual o menor de 3 en la escala EVA. Quedaron reflejadas en la recogida de datos asimismo las incidencias y efectos secundarios como aparición de bloqueo motor, parches analgésicos, necesidad de recolocación de catéter o complicaciones mayores neurológicas.

\section{RESULTADOS}

Los datos demográficos de la población estudiada (120 pacientes) se muestran en la Tabla I.

TABLA I. DATOS DEMOGRÁFICOS DE LOS PACIENTES

\begin{tabular}{lc}
\hline & Media $\pm D E$ \\
\cline { 2 - 2 } Edad (años) & $28,26 \pm 6,65$ \\
Peso $(\mathrm{kg})$ & $82,68 \pm 9,63$ \\
Talla $(\mathrm{cm})$ & $161,16 \pm 4,49$ \\
IMC $\left(\mathrm{kg} / \mathrm{m}^{2}\right)$ & $32 \pm 3,44$ \\
\hline
\end{tabular}

El hábito corporal de las pacientes, los antecedentes de punción epidural dificultosa, los signos de escoliosis y las referencias anatómicas, junto con la clasificación según grado de anatomía espinal en relación con las punciones consideradas dificultosas según los criterios del estudio, se reflejan en las siguientes tablas (Tablas II-VI).

TABLA II. HÁBITO CORPORAL DE LAS PACIENTES INCLUIDAS EN EL ESTUDIO

\begin{tabular}{lccc}
\hline \multicolumn{1}{c}{$\begin{array}{c}\text { Hábito } \\
\text { corporal }\end{array}$} & $\begin{array}{c}N^{o} \\
\text { pacientes }\end{array}$ & $\begin{array}{c}\text { No } \\
\text { dificultosa }\end{array}$ & Dificultosa \\
\hline $\begin{array}{l}\text { Normal } \\
(I M C<30)\end{array}$ & 48 & $46 / 95,83 \%$ & $2 / 4,16 \%$ \\
$\begin{array}{l}\text { Obeso } \\
(I M C>30)\end{array}$ & 72 & $36 / 50 \%$ & $36 / 50 \%$ \\
\hline
\end{tabular}

TABLA III. ANTECEDENTES DE ANESTESIA ESPINAL CONSIDERADA DIFICULTOSA POR LAS PACIENTES

\begin{tabular}{lccc}
\hline $\begin{array}{c}\text { Antecedentes } \\
\text { de punción } \\
\text { dificultosa }\end{array}$ & $\begin{array}{c}N^{o} \\
\text { pacientes }\end{array}$ & $\begin{array}{c}\text { No } \\
\text { dificultosa }\end{array}$ & Dificultosa \\
\hline Si $i$ & 14 & $10 / 71,42 \%$ & $4 / 28,57 \%$ \\
No & 106 & $90 / 84,90 \%$ & $16 / 15,09 \%$ \\
\hline
\end{tabular}

TABLA IV. FRECUENCIA DE IDENTIFICACIÓN DE LAS CRESTAS ILIACAS EN LA INSPECCIÓN ANATÓMICA

\begin{tabular}{lccc}
\hline $\begin{array}{c}\text { Palpación de } \\
\text { las crestas } \\
\text { iliacas }\end{array}$ & $\begin{array}{c}N^{o} \\
\text { pacientes }\end{array}$ & $\begin{array}{c}\text { No } \\
\text { dificultosa }\end{array}$ & Dificultosa \\
\hline Sí & 72 & $58 / 80,55 \%$ & $14 / 19,44 \%$ \\
No & 48 & $22 / 45,83 \%$ & $26 / 54,16 \%$ \\
\hline
\end{tabular}


TABLA V. FRECUENCIA DE IDENTIFICACIÓN DE SIGNOS DE ESCOLIOSIS EN LA INSPECCIÓN ANATÓMICA

\begin{tabular}{lccc}
\hline $\begin{array}{c}\text { Signos de } \\
\text { escoliosis en } \\
\text { la inspección }\end{array}$ & $\begin{array}{c}N^{o} \\
\text { pacientes }\end{array}$ & $\begin{array}{c}\text { No } \\
\text { dificultosa }\end{array}$ & Dificultosa \\
\hline Si & 48 & $32 / 66,66 \%$ & $16 / 33,33 \%$ \\
No & 96 & $72 / 75 \%$ & $24 / 25 \%$ \\
\hline
\end{tabular}

TABLA VI. ESTADIAJE DE LAS PACIENTES SEGÚN LA CLASIFICACIÓN DE CHIEN

\begin{tabular}{lccc}
\hline $\begin{array}{c}\text { Grado } \\
\text { anatómico }\end{array}$ & $\begin{array}{c}N^{o} \\
\text { pacientes/ } \\
\%\end{array}$ & $\begin{array}{c}\text { No } \\
\text { dificultosa }\end{array}$ & Dificultosa \\
\hline Grado 1 & $39 / 32,5$ & $39 / 100 \%$ & $0 / 0 \%$ \\
Grado 2 & $22 / 18,33$ & $14 / 63,63 \%$ & $6 / 27,27 \%$ \\
Grado 3 & $38 / 31,66$ & $24 / 63,15 \%$ & $14 / 36,84 \%$ \\
Grado 4 & $20 / 16,6$ & $2 / 10 \%$ & $18 / 90 \%$ \\
& & TOTAL & TOTAL \\
& & $76 / 63,33 \%$ & $48 / 36,67 \%$ \\
\hline
\end{tabular}

La mayoría de los catéteres epidurales fueron colocados con éxito en el primer intento, siendo calificados de punción difícil, según los criterios del estudio, el 36,67 \% de los casos. En la mayoría de los casos la técnica fue efectiva obteniendo una adecuada analgesia. La incidencia de repunción epidural fue del $5 \%$ (6 casos) por motivo de analgesia insatisfactoria pese a la retirada parcial del catéter y refuerzos con bolos de fármaco anestésico por catéter, mientras que la incidencia de analgesia incompleta que no precisó repunción fue del 11,66 \% (11 casos).

En general la tasa de éxito en el primer intento fue de $66,33 \%$, siendo el factor más influyente la calidad de los puntos de referencia anatómicos. En las pacientes clasificadas en el grado 4 de nuestro estudio, la tasa de punción epidural considerada dificultosa supuso un $90 \%$, necesitando en el $80 \%$ de esos casos 3 o más intentos. La incidencia de punción dificultosa encontrada en este estudio es del $36,84 \%$ en las del grupo 3 , del $27,27 \%$ en las clasificadas en el grupo 2 y del $0 \%$ en las del grupo 1 . En las pacientes en que la inspección de la espalda resultaba sospechosa de escoliosis o deformidades, la tasa de punción dificultosa ascendió a 33,33 \% (16 pacientes).

En las pacientes con antecedentes personales de técnica neuroaxial dificultosa se encontró dificultad de punción epidural en un $28,57 \%$ de los casos.

El número de intentos según los factores predictores de dificultad se expone en las siguientes tablas (Tablas VII-IX):
TABLA VII. NÚMERO DE INTENTOS EN RELACIÓN CON LA CLASIFICACION DE CHIEN

\begin{tabular}{lcccc}
\hline & $\begin{array}{c}1 \\
\text { intento }\end{array}$ & 2 intentos & $\begin{array}{c}3 \geq \\
\text { intentos }\end{array}$ & Total \\
\hline Grupo 1 & $39 / 100 \%$ & $0 / 0 \%$ & $0 / 0 \%$ & 39 \\
Grupo 2 & $14 / 63,63 \%$ & $6 / 27,27 \%$ & $0 / 0 \%$ & 22 \\
Grupo 3 & $24 / 63,15 \%$ & $8 / 21,05 \%$ & $6 / 15,78 \%$ & 38 \\
Grupo 4 & $2 / 10 \%$ & $2 / 10 \%$ & $16 / 80 \%$ & 20 \\
\hline
\end{tabular}

TABLA VIII. NÚMERO DE INTENTOS SEGÚN SIGNOS DE ESCOLIOSIS EN LA INSPECCIÓN

\begin{tabular}{lcccc}
\hline $\begin{array}{l}\text { Signos de } \\
\text { escoliosis } \\
\text { en la } \\
\text { inspección }\end{array}$ & 1 intento & 2 intentos & $\begin{array}{c}3 \geq \\
\text { intentos }\end{array}$ & Total \\
\hline Si & $32 / 66,66 \%$ & $10 / 20,83 \%$ & $6 / 12,50 \%$ & 48 \\
No & $72 / 75 \%$ & $12 / 12,50 \%$ & $12 / 12,50 \%$ & 96 \\
\hline
\end{tabular}

TABLA IX. NÚMERO DE INTENTOS SEGÚN IDENTIFICACIÓN DE CRESTAS ILIACAS

\begin{tabular}{lcccc}
\hline $\begin{array}{l}\text { Palpación } \\
\text { de crestas } \\
\text { iliacas }\end{array}$ & $\begin{array}{c}1 \\
\text { intento }\end{array}$ & $\begin{array}{c}2 \\
\text { intentos }\end{array}$ & $\begin{array}{c}3 \geq \\
\text { intentos }\end{array}$ & Total \\
\hline Si & $58 / 80,55 \%$ & $10 / 13,88 \%$ & $4 / 5,55 \%$ & 72 \\
No & $22 / 45,83 \%$ & $6 / 12,50 \%$ & $20 / 41,66 \%$ & 48 \\
\hline
\end{tabular}

Destaca del análisis de los datos recogidos en el presente estudio que el hábito corporal (normal u obesa si el IMC es mayor de 35) no tuvo efecto significativo sobre la tasa de éxito con el primer intento, siendo la incidencia de punción dificultosa del $50 \%$ en las pacientes con IMC $\geq$ 30. Tampoco tuvo efecto sobre el éxito de la punción en el primer intento el factor demográfico de la edad.

\section{DISCUSIÓN}

El presente estudio representa una buena estimación de la dificultad técnica de la técnica epidural en la paciente obstétrica; teniendo en cuenta la seguridad del procedimiento y el posible traumatismo de estructuras anatómicas o complicaciones derivadas de la misma, creemos que la experiencia del anestesiólogo en técnicas de bloqueo neuroaxial da lugar a que estos procedimientos se realicen de forma menos traumática, hecho que ha sido anteriormente observado en algunos estudios publicados $(7,8)$.

Es importante enfatizar la importancia de evitar los procedimientos traumáticos cuando realizamos técnicas de 
Rev. Soc. Esp. del Dolor, Vol. 20, N. ${ }^{\text { 5 }}$, Septiembre-Octubre 2013

bloqueo espinal, sobre todo cuando los pacientes tienen alteraciones de la coagulación o están siendo tratados con medicaciones que alteran la agregación plaquetaria o afectan a la función de los factores de la coagulación. Este hecho es importante porque muchas de las gestaciones que son consideradas de alto riesgo son vigiladas por trombofilias filiadas en la consulta del tocólogo tras abortos de repetición y precisan tratamiento durante la gestación con antiagregantes o heparinas, y esto no es una contraindicación para la indicación de anestesia epidural durante el trabajo de parto (9).

Nos parece interesante el hecho de reconocer factores maternos que intenten predecir cuándo puede ser complicada la colocación de un catéter epidural en una paciente obstétrica para prestar mayor atención durante el proceso e incluso intentar la búsqueda de una persona experta para que realice la técnica con mayor seguridad en casos de alto riesgo de producir lesiones traumáticas. Han sido publicadas diversas escalas para predecir la dificultad para las prácticas de bloqueos epidurales y subaracnoideos, y varias concluyen que son los releves cutáneos de las estructuras óseas los predictores mas importantes de una técnica neuroaxial dificultosa, sin encontrar diferencias significativas entre anestesiólogos con o sin experiencia probablemente porque no se trata de pacientes obstétricas con peculiaridades con respecto a pacientes programados para intervenciones quirúrgicas $(8,10,11)$.

En todas las pacientes de nuestro estudio la técnica epidural fue realizada en posición de sedestación dado que es el protocolo de nuestro centro, y sería difícil saber si los datos reflejados serían extrapolables a la realización de la técnica en decúbito lateral, donde los puntos de referencia anatómicos son más difíciles de identificar. Destaca del análisis de los datos recogidos en el presente estudio que el hábito corporal (normal u obesa si el IMC es mayor de $35)$ no tuvo efecto significativo sobre la tasa de éxito con el primer intento, siendo la incidencia de punción dificultosa del $50 \%$ en las pacientes con IMC $\geq 30$. Tampoco tuvo efecto sobre el éxito de la punción en el primer intento el factor demográfico de la edad.

\section{CONCLUSIONES}

Nuestra recogida de datos de 120 procedimientos en pacientes obstétricas con trabajo de parto concluye que el método más fiable para determinar de antemano la posi- bilidad de una dificultad técnica de colocación del catéter epidural lumbar es un examen de la espalda de la paciente para identificar la calidad de los puntos de referencia anatómicos e identificar la deformidad obvia de la columna vertebral. El hábito corporal es menos fiable y los antecedentes referidos por la paciente de técnica anteriormente dificultosa se relacionan con la posibilidad de dificultades técnicas en posteriores colocaciones de epidural lumbar.

CORRESPONDENCIA:

Luisa María Charco Roca

e-mail: latinloisii@hotmail.com

\section{BIBLIOGRAFÍA}

1. Vandermeulen E, van Aken H, Vermylen J. Anticoagulants and spinal epidural anesthesia. Anesth Analg 1994;79(6):1165-77.

2. Auroy Y, Narchi P, Messiah A, Litt L, Rouvier B, Samii K. Serious complications related to regional anesthesia. Anesthesiology 1997;87(3):479-86.

3. Renck H. Neurological complications of central nerve blockade. Acta Anaesthesiol Scand 1995;39(7):859-68.

4. Konrad C, Schupfer G, Wietlisbach M, Gerber H. Learning manual skills in anaesthesiology: Is there a recommended number of cases for anesthetic procedures? Anesth Analg 1998;86(3):635-9.

5. De Oliveira Filho GR. The construction of learning curves for basic skills in anesthetic procedures: An application for the cumulative sum method. Anesth Analg 2002;95(2):411-6.

6. Sprung J, Bourke DL, Grass J, Hammel J, Mascha E, Thomas $P$ et al. Predicting the difficult neuraxial block: A prospective study. Anesth Analg 1999;89(2):384-9.

7. M. Palencia, E. Guasch, D. Navas, F. Gilsanz. Factores asociados a la dificultad de punción epidural en analgesia obstétrica. Rev Esp Anestesiol Reanim 2006;53:139-44.

8. Chien I, Lu IC, Wang FY, Soo LY, Yu KL, Tang CS. Spinal process landmark as a predicting factor for difficult epidural block: A prospective study in Taiwanese patients. Kaohsiung J Med Sci 2003;19(11):563-8.

9. Horlocker TT, Wedel DJ. Anticoagulants, antiplatelet therapy and neuraxial blockade. Anesth Clin North Am 1992;10:111.

10. Atallah MM, Demian AD, Shorrab AA. Development of a difficulty score for spinal anesthesia. Br J Anaesth 2004;92(3):354-60.

11. Karraz M. Four grades score to predict the difficulty of epidural block. Int J Obstet Anaesth 2003;12(S1):37. 